\title{
Plasma C4d as marker for lupus nephritis in systemic lupus erythematosus
}

\author{
Myriam Martin ${ }^{1}$, Karolina I. Smoląg ${ }^{1}$, Albin Björk', Birgitta Gullstrand ${ }^{2}$, Marcin Okrój ${ }^{3}$, Jonatan Leffler ${ }^{4}$, \\ Andreas Jönsen², Anders A. Bengtsson ${ }^{2}$ and Anna M. Blom ${ }^{*}$
}

\begin{abstract}
Background: In the present study, we sought to evaluate the complement activation product C4d as a marker for lupus nephritis in systemic lupus erythematosus (SLE).

Methods: C4d levels were determined by enzyme-linked immunosorbent assay in plasma samples of patients with established SLE using a novel approach based on detection of a short linear cleavage neoepitope. Cross-sectional associations were studied in 98 patients with SLE with samples taken at lower or higher respective disease activity. Temporal associations were investigated in 69 patients with SLE who were followed longitudinally for up to 5 years. Plasma samples from 77 healthy donors were included as controls.

Results: C4d levels were negligible in healthy control subjects and significantly increased in patients with SLE in the cross-sectional study $(p<0.0001)$. C4d levels discriminated between higher and lower disease activity according to ROC curve analysis ( $p<0.001$ ), exhibiting a positive predictive value of $68 \%$. At higher disease activity, C4d levels correlated with the modified Systemic Lupus Erythematosus Disease Activity Index $(p=0.011)$ and predominantly with lupus nephritis ( $p=0.003$ ), exhibiting a sensitivity of $79 \%$ to identify patients with nephritis. High C4d levels together with the presence of anti-dsDNA autoantibodies preceded and thus predicted future lupus nephritis in the longitudinal study (OR 5.4, 95\% Cl 1.4-21.3). When we considered only patients with renal involvement (19 of 69) during the longitudinal study, we found that high $\mathrm{C} 4 \mathrm{~d}$ levels alone could forecast recurrence of future lupus nephritis (OR 3.3, 95\% Cl 1.2-9.6).
\end{abstract}

Conclusions: C4d appears to be a valuable marker for use in monitoring of patients with SLE, particularly for lupus nephritis. Importantly, C4d levels can predict impending flares of lupus nephritis and may thus be useful for informing treatment.

Keywords: Systemic lupus erythematosus, Complement, C4d, Flare, Lupus nephritis

\section{Background}

Systemic lupus erythematosus (SLE) is a chronic autoimmune disorder with complex etiology and multiorgan involvement causing clinical manifestations such as malar rash, arthritis, and renal disorders [1]. It is characterized by periods of illness and flares, which result in reduced quality of life and increased mortality as well as periods of low disease activity. Lupus nephritis (LN) is one of the most severe manifestations of SLE. There is no curative treatment

\footnotetext{
* Correspondence: anna.blom@med.lu.se

${ }^{1}$ Department of Translational Medicine, Section of Medical Protein Chemistry, Lund University, Inga Marie Nilsson's Street 53, 20502 Malmö, Sweden
} Full list of author information is available at the end of the article for SLE, and current treatment strategies are aimed at minimizing organ damage.

Activation of the complement system is a hallmark of SLE. Low serum levels of complement components C3 and $\mathrm{C} 4$ have been used for over 50 years to indicate lupus activity and are included in the Systemic Lupus Erythematosus Disease Activity Index (SLEDAI). Because levels of C3 or C4 depend not only on complement activation but also on the rate of synthesis, the measurement of complement activation products has been suggested as a more specific SLE biomarker [2-4]. A variety of other biomarkers are used to diagnose SLE, to monitor disease activity, and to identify and/or predict specific organ involvement [5]. However, no 
biomarker covers all aspects of the different phenotypes of the disease, and some assays are prone to producing false-positive data owing to mistreatment of the samples.

In the present study, by applying our novel, robust, and feasible C4d enzyme-linked immunosorbent assay (ELISA), we show that C4d, which is the final cleavage product of $\mathrm{C} 4$ arising during complement activation, is a valuable marker to discriminate higher disease activity and especially LN. C4d levels correlate with SLEDAI and can forecast impending renal flares in patients with previous renal involvement.

\section{Methods}

\section{Study participants}

Patients with SLE

All patients were recruited from the Department of Rheumatology, Lund University, Lund, Sweden, and fulfilled at least four of the American College of Rheumatology classification criteria for SLE [6], which are listed together with demographics and treatments in Table 1. The cross-sectional study group included 98 patients with SLE whose disease activity was assessed prospectively using the Systemic Lupus Erythematosus Disease Activity Index 2000 (SLEDAI-2K) [7]. Two plasma samples were selected for each patient and collected at time points with higher and lower relative clinical disease activity. The longitudinal study group encompassed 69 patients followed periodically with a median (range) of 13 (2-42) visits. A time interval of 70 \pm 30 days was chosen to assess temporal associations. Fifty-four patients overlapped in both study groups. However, the sampling time points did not overlap. Disease activity was recorded at every visit using the SLEDAI-2K. Samples were defined as nephritis-positive when SLEDAI scores were given for any of the following: urinary cast, hematuria, proteinuria, or pyuria. Blood samples were centrifuged $1 \mathrm{~h}$ after sampling, and plasma was aliquoted and stored at -80 ${ }^{\circ} \mathrm{C}$ no longer than $2 \mathrm{~h}$ after venipuncture. Although we do not have genetic data for a majority of the patients, they were all screened for complement deficiency using functional tests. These showed that none of the patients had a complete deficiency in C3 or C4. However, we cannot rule out partial deficiencies.

\section{Healthy control subjects}

Seventy-seven healthy control subjects were recruited in 2011 at the Department of Translational Medicine, Lund University, Malmö, Sweden. Fifty-two (68\%) patients were female, and their median age was 38 years (range

Table 1 Demographics and clinical characteristics of the cross-sectional and longitudinal study groups

\begin{tabular}{|c|c|c|}
\hline Characteristics & Study $1(n=98)$ Cross-sectional Higher/lower disease activity & Study $2(n=69)$ Longitudinal \\
\hline Age at sampling, years, median (range) & $41(14-75) / 43(19-81)$ & $39.2(18-76)$ \\
\hline Female sex, $n(\%)$ & $88(91)$ & $63(91.3)$ \\
\hline Disease duration, years, median (range) & $4(0-40) / 7(0-43)$ & $7.5(0-41)$ \\
\hline Follow-up duration, days, median (range) & N/A & $778(139-1792)$ \\
\hline SLEDAI-2K & $9(2-28) / 2(0-12)$ & $2(0-24)$ \\
\hline \multicolumn{3}{|l|}{ ACR classification criteria, $n$} \\
\hline Malar rash & 65 & 46 \\
\hline Discoid rash & 29 & 19 \\
\hline Photosensitivity & 61 & 43 \\
\hline Oral ulcer & 29 & 24 \\
\hline Arthritis & 82 & 60 \\
\hline Serositis & 56 & 39 \\
\hline Lupus nephritis & 45 & 34 \\
\hline Neurological disorder & 8 & 3 \\
\hline Hematological disorder & 53 & 38 \\
\hline Immunological disorder & 74 & 53 \\
\hline Antinuclear antibodies & 96 & 69 \\
\hline \multicolumn{3}{|l|}{ Treatment, $n$} \\
\hline Antimalarial & $36 / 35$ & 46 \\
\hline Corticosteroid & $64 / 64$ & 55 \\
\hline Immunomodulatory & $42 / 51$ & 41 \\
\hline
\end{tabular}


21-74). Plasma samples were prepared according to the same protocol used for the patients' samples.

\section{ELISA detecting soluble C4d}

Nunc MaxiSorp 96-well plates (Invitrogen, Carlsbad, CA, USA) were coated with rabbit anti-human C4d neoepitope-specific antibody [8]. After quenching with washing buffer $(50 \mathrm{mM}$ Tris- $\mathrm{HCl}, 0.15 \mathrm{M} \mathrm{NaCl}, 0.1 \%$ Tween, $\mathrm{pH}$ 7.5) supplemented with $3 \%$ fish gelatin (Norland Products, Cranbury, NJ, USA), patient or control plasma and pooled plasma from healthy volunteers (lacking C4d) supplemented with Escherichia coli-expressed C4d standard in serial dilutions were diluted to $4 \%$ in PBS with $0.02 \%$ Tween-20 and $0.02 \mathrm{M}$ disodium ethylenediaminetetraacetate dihydrate and added to the plate. Detection was achieved using mouse anti-human C4d antibody (catalogue number 253; Quidel, San Diego, CA, USA) followed by horseradish peroxidase-conjugated goat anti-mouse secondary antibody (Dako, Carpinteria, CA, USA). Plates were developed using $o$-phenylenediamine dihydrochloride as a substrate, and absorbance was measured at $490 \mathrm{~nm}$ using a Varian Cary 50 microplate reader (Agilent Technologies, Santa Clara, CA, USA). The lowest detection limit was $5.6 \mu \mathrm{g} / \mathrm{L}$. Values below the detection limit were set to $0.001 \mathrm{mg} / \mathrm{L}$ for statistical calculations. The inter-assay coefficient of variation was $16.7 \%$, and the intra-assay coefficient of variation was $13.2 \%$.

\section{Standard laboratory tests and reference values}

All routine laboratory tests necessary to assess disease activity were executed at the Unit of Clinical Immunology (Lund, Sweden). Until 2009, levels of C3 and C4 were measured with the ABX Pentra 400 assay (Horiba Medical, Irvine, CA, USA) using antihuman C3c (catalogue number Q0368; Dako) and antihuman C4 (catalogue number Q0369; Dako) antibodies. Thereafter, the IMMAGE 800 system (Beckman Coulter, Brea, CA, USA) was applied for nephelometry using antihuman C3 (catalogue number 446450; Beckman Coulter) and antihuman C4 (catalogue number 446490; Beckman Coulter) antibodies. A modified version of the SLEDAI 2000 (mSLEDAI), excluding scores for low levels of complement factors and/or anti-doublestranded DNA (dsDNA) antibodies, was also calculated. To create categorized variables, the following clinical routine reference values were applied: low $\mathrm{C} 3<0.77 \mathrm{~g} / \mathrm{L}$ and low $\mathrm{C} 4<0.12 \mathrm{~g} / \mathrm{L}$.

\section{Statistical analyses}

Statistical significance for nonparametric continuous data was calculated using the Mann-Whitney $U$ test for two groups and the Kruskal-Wallis rank-sum test for more than two groups. Data are presented as medians with $25-75 \%$ quantiles plus whiskers. Correlations of nonparametric data were analyzed using Spearman's rank-order correlation test. For some analyses, C4d levels were categorized according to ROC curve analyses (cross-sectional study $0.39 \mathrm{mg} / \mathrm{L}$ for higher disease activity and $0.42 \mathrm{mg} / \mathrm{L}$ for $\mathrm{LN}$; longitudinal study $1.1 \mathrm{mg} / \mathrm{L}$ for LN). ROC curves and AUCs, together with positive predictive values (PPVs) and negative predictive values (NPVs), were determined to evaluate accuracy. McNemar's test was used for comparison of two markers in terms of accuracy. ORs and 95\% CIs were calculated to estimate the relative risk. A generalized estimating equation was used when estimating ORs or regression coefficients in models with correlated outcomes, which can arise when the same patient is included more than once. A statistical significance level of ( $p$ value) $<0.05$ was defined as statistically significant. Analyses were carried out using JMP Pro 12 (SAS Institute, Cary, NC, USA) and IBM SPSS Statistics 23 (IBM, Armonk, NY, USA) software.

\section{Results}

C4d levels were increased in patients with SLE and associated strongly with higher disease activity in crosssectional study

C4d levels were measured with our in-house C4d ELISA [8]. In 63 of the 77 healthy control samples, C4d levels were below the detection limit. The level in the remaining 14 samples ranged from $0.005 \mathrm{mg} / \mathrm{L}$ to $0.138 \mathrm{mg} / \mathrm{L}$ (Fig. 1a). In patients with SLE, C4d levels were significantly increased, both in lower $(0.17 \mathrm{mg} / \mathrm{L}, 0.001-0.51 \mathrm{mg} / \mathrm{L})$ and in higher (0.49 mg/L, 0.08-1.31 mg/L) disease activity (Fig. 1a). Paired analysis of each patient revealed that C4d levels, on average, significantly increased threefold between lower and higher disease activity $(p<0.0001)$, whereas $\mathrm{C} 3$ levels decreased only 1.1-fold (lower activity $0.88 \mathrm{~g} / \mathrm{L}, 0.72-1.05 \mathrm{~g} / \mathrm{L}$; higher activity $0.79 \mathrm{~g} / \mathrm{L}, 0.64-1.01 \mathrm{~g} / \mathrm{L} ; p=0.028)$ and $\mathrm{C} 4$ levels even insignificantly (lower activity $0.17 \mathrm{~g} / \mathrm{L}, 0.13-0.21 \mathrm{~g} / \mathrm{L}$; higher activity $0.16 \mathrm{~g} / \mathrm{L}, 0.11-0.21 \mathrm{~g} / \mathrm{L} ; p=0.108)$. Thus, the magnitude of change was higher for C4d than for C3 and C4. Sixty-two patients exhibited higher C4d levels at higher disease activity, 7 patients exhibited unaltered levels, and 29 patients exhibited lower levels (Fig. 1b). C3 levels were lower in 59 patients at higher disease activity, unaltered in 1 patient, and higher in 38 patients. C4 levels were lower in 55 patients at higher disease activity, unchanged in 7 patients, and higher in 36 patients. As expected, at higher disease activity, C4d levels correlated negatively with C3 $\left(r_{\mathrm{s}}=-0.414\right.$, $p<0.0001)$ and C4 levels $\left(r_{\mathrm{s}}=-0.29, p<0.0038\right)$.

Medications differed between patients (Table 1), but no significant correlation with C4d levels and different therapies was observed.

\section{C4d was an accurate marker to discriminate between} higher and lower disease activity in cross-sectional study Area under the ROC curve analysis confirmed that C4d displays statistically significant accuracy as a marker to differentiate higher from lower disease activity, whereas C3 and C4 do not (Fig. 1c). C4d levels were categorized 

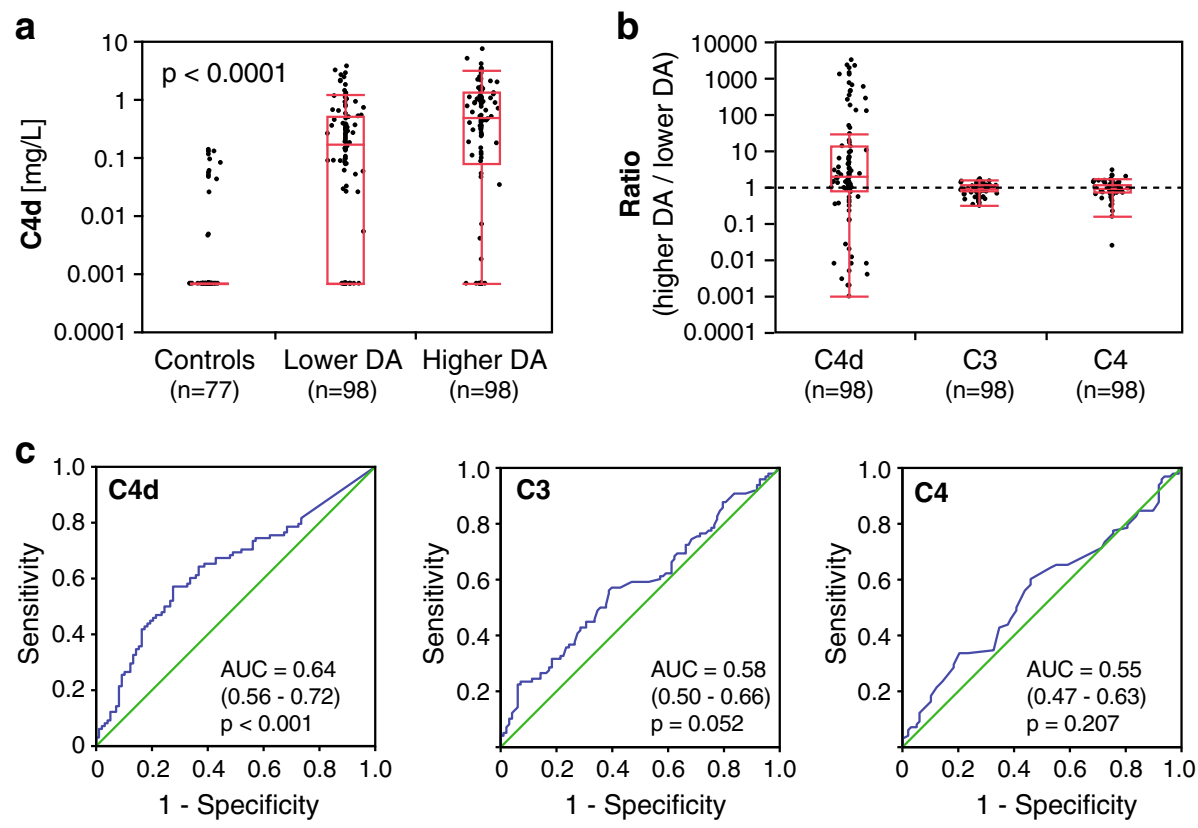

d

\begin{tabular}{l|l|l|l} 
& C4d & C3 & C4 \\
\hline Sensitivity [\%] & $57(47-67)$ & $49(39-59)$ & $30(21-40)$ \\
\hline Specificity [\%] & $73(63-81)$ & $65(55-75)$ & $82(73-89)$ \\
\hline PPV [\%] & $68(59-75)$ & $59(50-67)$ & $62(49-73)$ \\
\hline NPV [\%] & $63(57-69)$ & $56(50-62)$ & $54(50-58)$
\end{tabular}

Fig. 1 Plasma C4d levels are increased in patients with systemic lupus enythematosus (SLE) in the cross-sectional study, and C4d is convincing as an accurate marker for identifying higher disease activity (DA). a C4d levels in healthy control subjects $(n=77)$ and patients with SLE at lower $(n=98)$ and higher $(n=98)$ DA. Data are presented as medians with $25-75 \%$ quantiles plus whiskers, and significance was calculated using the Kruskal-Wallis rank-sum test. b Ratio of higher DA to lower DA of C4d, C3, and C4 levels in patients with SLE $(n=98)$. The dashed line indicates equal levels at higher and lower DA. Data are presented as medians with 25-75\% quantiles plus whiskers. c Area under the ROC curve analysis shows that only C4d, but not C3 and C4, exhibits accuracy as a marker for higher DA. $\mathbf{d}$ Sensitivity, specificity, positive predictive value (PPV), and negative predictive value (NPV) for C4d, C3, and C4 as markers for higher DA. Data are presented with 95\% Cls

according to ROC curve, and C3 and C4 levels were categorized according to routine clinical reference values. C4d had the highest sensitivity (57\%), PPV (68\%), and NPV (63\%) as a marker for higher disease activity (Fig. 1d), whereas C4 exhibited the highest specificity (82\%). High C4d levels have statistically superior accuracy as a marker of higher disease activity compared with low C4 levels ( $p<0.0001$ by McNemar's test) and accuracy similar to low C3 levels $(p=0.345)$.

\section{C4d levels correlate with disease activity}

As anticipated, C4d levels were significantly associated with low complement and occurrence of anti-dsDNA antibodies (Table 2). Thus, the mSLEDAI, excluding scores for low levels of complement factors and anti-dsDNA antibodies, was applied. In the cross-sectional cohort, elevated levels of C4d were detected at higher disease activity and correlated significantly with mSLEDAI (Fig. 2a). As expected, levels of C3 and C4 inversely correlated with mSLEDAI. At lower disease activity, neither $\mathrm{C} 4 \mathrm{~d}$ nor $\mathrm{C} 3$ nor $\mathrm{C} 4$ levels correlated significantly with mSLEDAI. In the longitudinal study group, only C4d, but neither C3 nor C4 levels, correlated significantly with $\operatorname{mSLEDAI}(\beta=0.05, p=0.022)$.

\section{C4d levels correlated positively with LN in cross-sectional study}

LN is considered a severe manifestation in SLE, and it is strongly associated with complement activation caused by autoantibody deposition in the kidney [9]. In the higher disease activity group, 34 patients had LN. These patients exhibited significantly higher C4d levels than patients without LN (Fig. 3a, Table 2). C3 levels were significantly lower in patients with $\mathrm{LN}(0.67 \mathrm{~g} / \mathrm{L}, 0.48-0.82 \mathrm{~g} / \mathrm{L}, p<0.001)$ than in patients without LN $(0.91 \mathrm{~g} / \mathrm{L}, 0.71-1.11 \mathrm{~g} / \mathrm{L})$. Similar findings were obtained for $\mathrm{C} 4$ levels $(0.14 \mathrm{~g} / \mathrm{L}, 0.1-0.17$ $\mathrm{g} / \mathrm{L}$; versus $0.16 \mathrm{~g} / \mathrm{L}, 0.12-0.23 \mathrm{~g} / \mathrm{L} ; p=0.038)$.

The ROC curve analysis revealed that $\mathrm{C} 4 \mathrm{~d}$ as well as $\mathrm{C} 3$ and $\mathrm{C} 4$ levels all displayed statistically significant accuracy as markers for LN (Fig. 3b). C4d had the highest sensitivity (79\%), PPV (50\%), and NPV (84\%), whereas C4 exhibited 
Table 2 Systemic Lupus Erythematosus Disease Activity Index-qualifying symptoms and C4d levels at higher disease activity in the cross-sectional study $(n=98)$

\begin{tabular}{|c|c|c|c|c|c|c|}
\hline \multirow[b]{3}{*}{ Clinical features } & \multicolumn{5}{|c|}{ Higher disease activity } & \multirow{3}{*}{$\begin{array}{l}\text { Lower } \\
\text { disease } \\
\text { activity } \\
\text { No. of } \\
\text { subjects }\end{array}$} \\
\hline & \multicolumn{2}{|c|}{ Feature present } & \multicolumn{2}{|c|}{ Feature absent } & \multirow[b]{2}{*}{$p$ Value } & \\
\hline & $\begin{array}{l}\text { No. of } \\
\text { subjects }\end{array}$ & $\mathrm{C} 4 \mathrm{~d}(\mathrm{mg} / \mathrm{L})$ & $\begin{array}{l}\text { No. of } \\
\text { subjects }\end{array}$ & $\mathrm{C} 4 \mathrm{~d}(\mathrm{mg} / \mathrm{L})$ & & \\
\hline Seizure & 2 & $0.56(0-1.12)$ & 96 & $0.49(0.09-1.32)$ & - & 0 \\
\hline Psychosis & 0 & - & 98 & $0.49(0.08-1.31)$ & - & 0 \\
\hline Organic brain syndrome & 0 & - & 98 & $0.49(0.08-1.31)$ & - & 0 \\
\hline Visual disturbance & 7 & $0.53(0.35-0.88)$ & 91 & $0.45(0.05-1.32)$ & 0.658 & 0 \\
\hline Cranial nerve disorder & 0 & - & 98 & $0.49(0.08-1.31)$ & - & 0 \\
\hline Lupus headache & 2 & $0.39(0-0.77)$ & 96 & $0.49(0.09-1.32)$ & - & 0 \\
\hline Cerebrovascular accident & 2 & 0 & 96 & $0.51(0.10-1.32)$ & - & 0 \\
\hline Vasculitis & 12 & $0.73(0.33-1.44)$ & 86 & $0.46(0.05-1.16)$ & 0.342 & 0 \\
\hline Arthritis & 29 & $0.15(0.001-1.03)$ & 69 & $0.65(0.21-1.33)$ & 0.033 & 1 \\
\hline Myositis & 1 & $0.19(0.19-0.19)$ & 97 & $0.51(0.07-1.31)$ & - & 0 \\
\hline $\begin{array}{l}\text { Kidney involvement } \\
\text { (urinary cast, hematuria, proteinuria, or pyuria) }\end{array}$ & 34 & $0.89(0.44-1.56)$ & 64 & $0.34(0-1.12)$ & 0.003 & 1 \\
\hline Rash & 30 & $0.45(0.10-1.15)$ & 68 & $0.52(0.05-1.38)$ & 0.745 & 4 \\
\hline Alopecia & 7 & $0.33(0.03-0.76)$ & 91 & $0.51(0.09-1.32)$ & 0.365 & 1 \\
\hline Oral ulcers & 7 & $0.40(0.11-1.24)$ & 91 & $0.51(0.05-1.32)$ & 0.868 & 0 \\
\hline Pleurisy & 13 & $0.88(0.34-2.16)$ & 85 & $0.44(0.05-1.19)$ & 0.172 & 0 \\
\hline Pericarditis & 7 & $1.07(0.12-2.85)$ & 91 & $0.45(0.05-1.24)$ & 0.237 & 0 \\
\hline Low complement (C3 or C4) & 54 & $0.87(0.35-1.46)$ & 44 & $0.17(0-0.99)$ & 0.001 & 40 \\
\hline Anti-DNA antibodies & 46 & $1.02(0.49-1.51)$ & 52 & $0.16(0-0.73)$ & $<0.0001$ & 24 \\
\hline Fever & 6 & $1.18(0.76-2.82)$ & 92 & $0.45(0.05-1.26)$ & 0.069 & 0 \\
\hline Thrombocytopenia & 3 & $0.40(0.18-1.51)$ & 95 & $0.45(0.04-1.12)$ & - & 2 \\
\hline Leukopenia & 10 & $0.47(0.22-1.53)$ & 88 & $0.44(0.03-1.12)$ & 0.49 & 6 \\
\hline
\end{tabular}

C4d levels are presented as median (25-75\% quantile), and significance was calculated using the Mann-Whitney $U$ test. Significant $p$ Values are highlighted in bold. C4d levels were calculated only when more than five patients had the particular symptom at higher disease activity

the highest specificity (73\%) (Fig. 3c). High C4d levels had a statistically superior accuracy as a marker for nephritis than low C4 levels ( $p=0.002$ by McNemar's test) and accuracy similar to low C3 levels $(p=0.508)$.

ORs were calculated using generalized estimating equation analysis that corrected for the presence of two samples from each patient to further study these associations for the categorized variables. High C4d levels as well as low C3 levels associated significantly with LN (Fig. 3d). Because the presence of anti-dsDNA antibodies is known to associate with LN [10], we investigated their combined association with high C4d and low C3 levels and determined significant associations (Fig. 3d). Of all other mSLEDAI-qualifying symptoms, C4d levels were only significantly altered in arthritis (Table 2 ).

\section{C4d can predict $L N$ in the longitudinal study}

An optimal biomarker should predict future flares of specific disease manifestations in order to adjust treatment in time. Thus, using the longitudinal study group, we additionally analyzed whether C4d had the potential to predict future LN within the chosen time interval of $70 \pm 30$ days. Neither high C4d nor low C3 nor low C4 levels alone could predict LN in the whole cohort. However, together with the presence of anti-dsDNA autoantibodies, high C4d levels showed a significant probability to precede future LN $(p=0.016)$ (Fig. 4a). Low C3 levels in combination with the presence of anti-dsDNA autoantibodies also associated significantly with future $\operatorname{LN}(p=0.031)$, whereas no association was found for $\mathrm{C} 4$.

Patients with SLE who have once had LN have an increased risk for recurrence of renal flares and generally have a worse prognosis [11]. Therefore, it is important to monitor this patient group vigilantly. During the chosen time interval, 19 of 69 patients had LN on at least one sampling occasion. To study whether C4d could predict future $\mathrm{LN}$ in patients with previous occurrence of $\mathrm{LN}$, all available samples collected after at least 
Cross-sectional study - higher disease activity
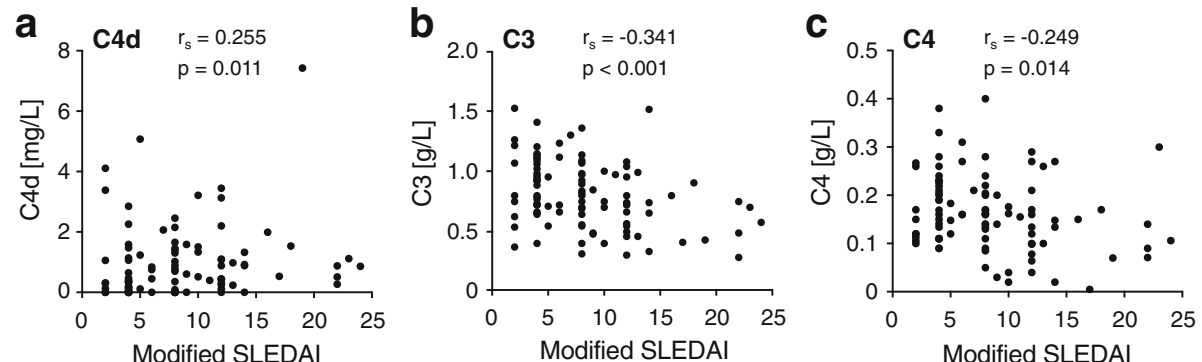

Fig. 2 C4d, C3, and C4 levels correlate with modified Systemic Lupus Erythematosus Disease Activity Index (SLEDAl). Correlation of C4d (a), C3 (b), and C4 (c) levels with modified SLEDAI (excluding scores for low levels of complement factors and/or anti-double-stranded DNA antibodies) in the cross-sectional study at higher disease activity. Significance and correlation coefficient $\left(r_{s}\right)$ were calculated using Spearman's rank-order correlation test

one occurrence of this manifestation were included (224 samples with 95 renal flares). Only C4d, but neither C3 nor $\mathrm{C} 4$, was associated significantly with future LN in the relapse group $(p=0.026)$ (Fig. 4b). High C4d levels alone could not predict any other mSLEDAIqualifying symptom in the longitudinal study group (data not shown).

\section{Discussion}

In this study, we report that $\mathrm{C} 4 \mathrm{~d}$, which is a proteolytic fragment of $\mathrm{C} 4$ generated exclusively upon complement activation, appears to be a valuable marker in the followup of SLE, in particular in LN. C4d levels correlate with disease activity and rise before renal flares, so that they have the potential to predict recurrence of LN.

\section{Cross-sectional study}

a Nephritis - higher DA

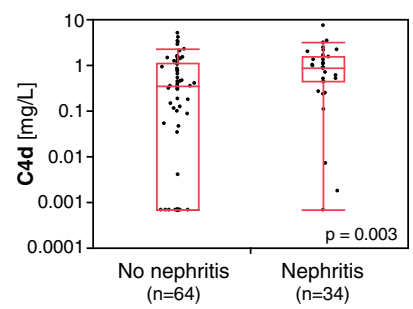

b Nephritis - higher DA

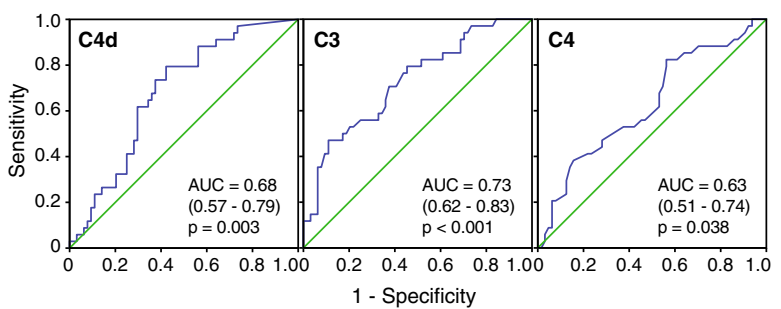

C

\begin{tabular}{l|l|l|l} 
& C4d & C3 & C4 \\
\hline Sensitivity [\%] & $79(62-91)$ & $71(53-85)$ & $41(25-59)$ \\
\hline Specificity [\%] & $58(45-70)$ & $63(50-74)$ & $73(59-84)$ \\
\hline PPV [\%] & $50(42-58)$ & $50(41-60)$ & $48(34-63)$ \\
\hline NPV [\%] & $84(73-91)$ & $80(70-87)$ & $71(64-77)$
\end{tabular}

d

Nephritis - complete cross-sectional cohort

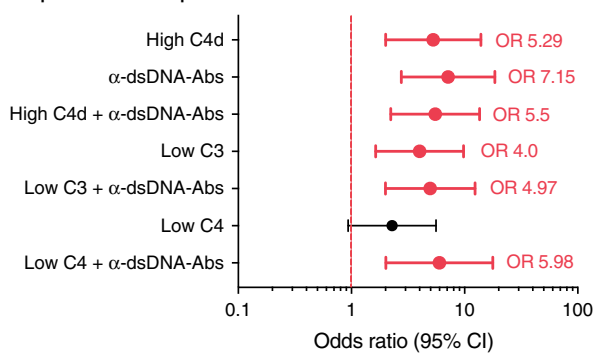

Fig. 3 Plasma C4d levels associate with lupus nephritis $(L N)$ in the cross-sectional study and are convincing as an accurate marker for $L N$. a At higher disease activity (DA), plasma C4d levels are elevated in patients with $L N(n=34)$ compared with patients without $L N(n=64)$. Data are presented as medians with 25-75\% quantiles plus whiskers, and significance was calculated using the Mann-Whitney $U$ test. b Area under the ROC curve analysis showing accuracy of C4d, C3, and C4 as markers for LN. c Sensitivity, specificity, positive predictive value (PPV), and negative predictive value (NPV) for C4d, C3, and C4 as markers for LN. Data are presented with 95\% Cls. d Association of high C4d (>0.42 mg/L), low C3 (<0.77 g/L), and low C4 $(<0.12 \mathrm{~g} / \mathrm{L})$ levels alone as well as in combination with the presence of anti-double-stranded DNA antibodies with LN. Significance was calculated using binary logistics, and ORs are indicated with a dot connected to the $95 \% \mathrm{Cl}$. Significant ORs are shown in bold and marked in red 


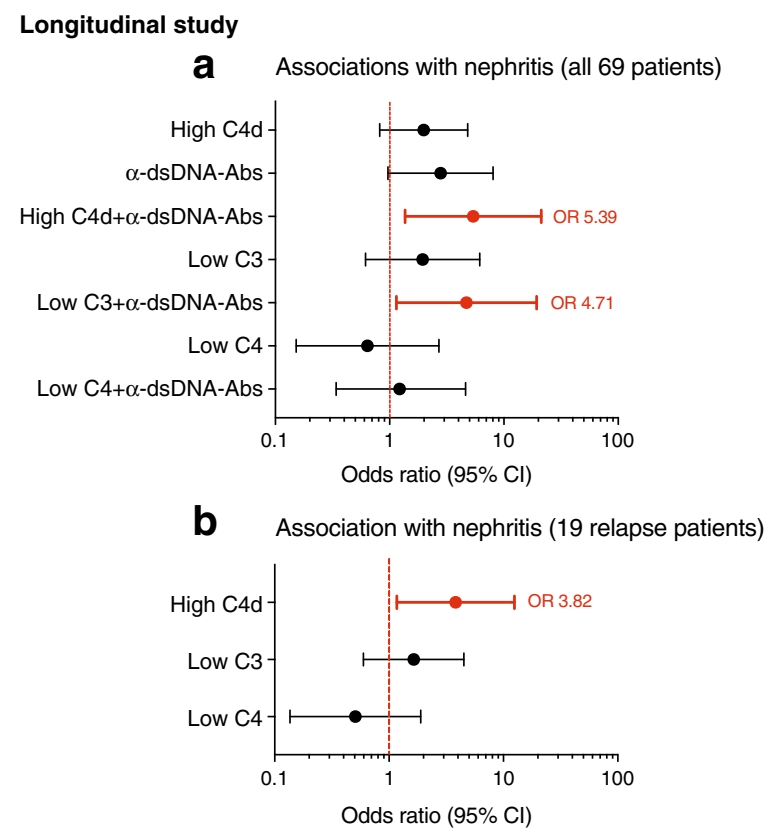

Fig. 4 Temporal association of $C 4 d, C 3$, and C4 with lupus nephritis in the longitudinal study. a Temporal association with $L N$ of high C4d (>1.1 mg/L), low C3 $(<0.77 \mathrm{~g} / \mathrm{L})$, and low C4 (<0.12 g/L) levels alone as well as in combination with the presence of anti-double-stranded DNA (anti-dsDNA) antibodies for all 69 patients within the chosen time interval of $70 \pm 30$ days. b Temporal association with LN for high C4d, low C3, and low C4 levels for the 19 relapse patients within the chosen time interval. Significance was calculated using a generalized estimating equation, and the ORs are indicated with a dot connected to the $95 \% \mathrm{Cl}$. Significant ORs are shown in bold and marked in red

Currently used C3 and C4 levels exhibit low sensitivity in follow-up of patients with SLE with broad reference intervals for healthy individuals [12, 13]. C3 and C4 levels are the net result of synthesis and consumption by activation, which are both elevated in inflammation. Accordingly, we observed only a moderate negative correlation of C3 and C4 with C4d, which occurs only as a product of complement activation. Furthermore, $\mathrm{C} 4 \mathrm{~d}$ levels correlated with mSLEDAI, in which the scores for low complement levels and anti-dsDNA antibodies levels were removed. The absence of $\mathrm{C} 4 \mathrm{~d}$ in healthy control subjects makes it per se a better marker, and the high fold change increase in higher disease activity, together with the fact that it is more accurate than $\mathrm{C} 4$ in discriminating higher disease activity, further supports its suitability. The fact that low complement is listed as a Systemic Lupus Collaborating Clinics classification criterion only to reflect the contribution of complement to SLE pathogenesis, but that it did not improve statistical modeling, further indicates that $\mathrm{C} 3$ and $\mathrm{C} 4$ are not optimal markers for monitoring SLE [13].

LN, which affects approximately $30-50 \%$ of patients with SLE, is one of the most devastating complications in SLE [14]. The pathogenesis of LN involves immune complex deposition [15], which strongly activates complement and gives rise to elevated levels of C4d. It is thus not surprising that $\mathrm{C} 4 \mathrm{~d}$ is a marker for nephritis in the cross-sectional study, but it is noteworthy that it has a better accuracy than $\mathrm{C} 4$ and similar accuracy to C3. Currently, there is still a need for a biomarker that would predict LN so that preventive measures could be initiated as soon as possible. Anti-dsDNA [10] and antinucleosome [16] antibodies were suggested as biomarkers for renal disease activity, but they were not convincing as sole biomarkers [17, 18]. C3/C4 levels alone were unable to forecast LN flares [11]. The determination of anti-C1q antibodies is a promising approach, and the measurement of co-occurring anti-Clq and antidsDNA antibodies seems most auspicious [19, 20]. In our longitudinal study, only 19 of 69 patients ever had LN during the chosen time interval, which makes it statistically challenging to investigate longitudinal associations. Because anti-dsDNA antibody levels have a certain potential to forecast LN, it was probable that the combination with $\mathrm{C} 4 \mathrm{~d}$ levels elevated the predictive power of $\mathrm{C} 4 \mathrm{~d}$. Patients who have already experienced LN have a high recurrence risk, and it is thus of utmost importance to recognize impending renal flares to avoid organ damage. Especially when considering the low patient numbers, it is striking that the sole measurement of $\mathrm{C} 4 \mathrm{~d}$ was statistically predictive for recurring $\mathrm{LN}$ flares in the relapse group. However, a larger cohort with more patients exhibiting renal disease is needed to confirm and strengthen this observation.

Up to $95 \%$ of patients with SLE can have arthritis, which is considered as a mild manifestation [21] not necessarily coinciding with complement activation. Arthritis was associated with low C4d levels, suggesting a different pathogenesis in arthritis in regard to complement activation. This opposing association was seen before in regard to decreased neutrophil extracellular trap degradation [22].

Measurement of $\mathrm{C} 4 \mathrm{~d}$, factor $\mathrm{Bb}$, and soluble C5b-9 was previously suggested for the assessment of disease activity and impending flares. Elevated C4d levels showed the highest sensitivity to precede flare [3]. Another study confirmed that $\mathrm{C} 4 \mathrm{~d}$ and factor $\mathrm{Bb}$ are sensitive indicators of moderate to severe lupus disease activity and suggested their measurement especially in patients who, despite evidence of clinical disease activity, do not exhibit altered C3 and C4 levels [4]. One problem with the commercially available C4d ELISA used in both studies is that it is much more prone to detect falsepositive readouts arising from sample handling, such as freezing and thawing. Our C4d ELISA has been proven to be much less prone to measure artifacts formed in the course of sample handling and to specifically determine the complement activation-specific cleaved form of 
C4d [8]. Furthermore, we have shown in a cohort of patients with leukemia treated with anti-CD20 antibodies that levels of C4d detected with our assay clearly correlated with other markers of complement activation, whereas the levels detected with the commercial assay did not [8]. This is due to the recognition of a very short linear neoepitope formed only after C4b cleavage to C4d instead of conformational neoepitopes, which apparently can be mimicked by nonproteolytic events. However, no direct comparison with a commercial assay was done for the patient samples used in the present study.

Another promising approach is the measurement of $\mathrm{C} 4 \mathrm{~d}$ bound to the surface of various blood cells, such as erythrocytes, reticulocytes, and platelets [23-26]. However, the drawback of this methodology is that it is work-intensive and requires advanced equipment. Because a significant amount of C4d remains soluble in plasma and body fluids [27], we are convinced that measurement by ELISA is more feasible in clinical diagnostics.

\section{Conclusions}

In summary, measuring C4d levels using our robust and practical assay provides a novel method of monitoring SLE activity that is at least as good as C3 and superior to $\mathrm{C} 4$ in identifying active disease and in particular LN. Most importantly, C4d levels are more valuable than either $\mathrm{C} 3$ or $\mathrm{C} 4$ in predicting recurrence of renal flares, aiding clinicians in adjusting treatment in time.

\section{Abbreviations}

ACR: American College of Rheumatology; DA: Disease activity; dsDNA: Double-stranded DNA; ELISA: Enzyme-linked immunosorbent assay; LN: Lupus nephritis; mSLEDAl: Modified Systemic Lupus Erythematosus Disease Activity Index; NPV: Negative predictive value; PPV: Positive predictive value; SLE: Systemic lupus erythematosus; SLEDAI: Systemic Lupus Erythematosus Disease Activity Index; SLEDAI-2K: Systemic Lupus Erythematosus Disease Activity Index 2000

\section{Acknowledgements}

The authors acknowledge all the patients and their physicians and thank Dr. Ben King for language revision.

\section{Funding}

The study was funded by the Swedish Research Council (2016-01142) Foundations of Österlund, Greta and Johan Kock, King Gustav V's 80th Anniversary Fund Foundation, Anna-Greta Crafoord, the Swedish Rheumatism Association, and clinical research grants for from ALF and the Skåne University Hospital. M.O. is supported by National Science Centre Poland (2014/14/E/NZ6/00182).

\section{Availability of data and materials}

The datasets used and/or analyzed during the present study are available from the corresponding author on reasonable request.

\section{Authors' contributions}

$M M, K I S K, A B, M O$, and $J L$ performed the experiments and analyzed the results. BG acquired and analyzed clinical parameters. $A A B$ and $A J$ recruited patients and supervised the study together with $M M$ and $A M B$, who also interpreted the data. MM drafted the manuscript together with AMB. All authors read, revised, and approved the final manuscript.

\section{Ethics approval and consent to participate}

Collection of samples for both cohorts and healthy control subjects was performed according to the Declaration of Helsinki and approved by the Regional Ethical Review Board in Lund, with all participants providing written consent to participate in the study.

\section{Consent for publication}

Not applicable.

\section{Competing interests}

$\mathrm{AMB}$ and $\mathrm{MO}$ are named as inventors in a patent application including claims to use of $\mathrm{C} 4 \mathrm{~d}$ as a biomarker. The other authors declare that they have no competing interests.

\section{Publisher's Note}

Springer Nature remains neutral with regard to jurisdictional claims in published maps and institutional affiliations.

\section{Author details}

'Department of Translational Medicine, Section of Medical Protein Chemistry, Lund University, Inga Marie Nilsson's Street 53, 20502 Malmö, Sweden.

${ }^{2}$ Rheumatology, Department of Clinical Sciences Lund, Lund University, Lund, Sweden. ${ }^{3}$ Department of Medical Biotechnology, Intercollegiate Faculty of Biotechnology, University of Gdańsk and Medical University of Gdańsk, Gdańsk, Poland. ${ }^{4}$ Telethon Kids Institute, University of Western Australia, Perth, Australia.

Received: 9 June 2017 Accepted: 10 November 2017

Published online: 06 December 2017

\section{References}

1. Yu C, Gershwin ME, Chang C. Diagnostic criteria for systemic lupus erythematosus: a critical review. J Autoimmun. 2014;48-49:10-3.

2. Illei GG, Tackey E, Lapteva L, Lipsky PE. Biomarkers in systemic lupus erythematosus: II. Markers of disease activity. Arthritis Rheum. 2004; 50(7):2048-65.

3. Buyon JP, Tamerius J, Belmont HM, Abramson SB. Assessment of disease activity and impending flare in patients with systemic lupus erythematosus: comparison of the use of complement split products and conventional measurements of complement. Arthritis Rheum. 1992;35(9):1028-37.

4. Manzi S, Rairie JE, Carpenter AB, Kelly RH, Jagarlapudi SP, Sereika SM, Medsger Jr TA, Ramsey-Goldman R. Sensitivity and specificity of plasma and urine complement split products as indicators of lupus disease activity. Arthritis Rheum. 1996:39(7):1178-88.

5. Liu CC, Ahearn JM. The search for lupus biomarkers. Best Pract Res Clin Rheumatol. 2009;23(4):507-23.

6. Tan EM, Cohen AS, Fries JF, Masi AT, McShane DJ, Rothfield NF, Schaller JG, Talal N, Winchester RJ. The 1982 revised criteria for the classification of systemic lupus erythematosus. Arthritis Rheum. 1982;25(11):1271-7.

7. Gladman DD, Ibañez D, Urowitz MB. Systemic lupus erythematosus disease activity index 2000. J Rheumatol. 2002;29(2):288-91.

8. Blom AM, Osterborg A, Mollnes TE, Okroj M. Antibodies reactive to cleaved sites in complement proteins enable highly specific measurement of soluble markers of complement activation. Mol Immunol. 2015;66(2):164-70.

9. Bao L, Cunningham PN, Quigg RJ. Complement in lupus nephritis: new perspectives. Kidney Dis (Basel). 2015;1(2):91-9.

10. Smeenk R, Duin T, Aarden L. Influence of $\mathrm{pH}$ on the detection of low- and high-avidity anti-dsDNA. J Immunol Methods. 1982;55(3):361-73.

11. Birmingham DJ, Irshaid F, Nagaraja HN, Zou X, Tsao BP, Wu H, Yu CY, Hebert $\mathrm{LA}$, Rovin BH. The complex nature of serum C3 and C4 as biomarkers of lupus renal flare. Lupus. 2010;19(11):1272-80.

12. Ritchie RF, Palomaki GE, Neveux LM, Navolotskaia O, Ledue TB, Craig WY Reference distributions for complement proteins C3 and C4: a practical, simple and clinically relevant approach in a large cohort. J Clin Lab Anal. 2004;18(1):1-8.

13. Petri M, Orbai AM, Alarcon GS, Gordon C, Merrill JT, Fortin PR, Bruce IN, Isenberg D, Wallace DJ, Nived O, et al. Derivation and validation of the Systemic Lupus International Collaborating Clinics classification criteria for systemic lupus erythematosus. Arthritis Rheum. 2012;64(8):2677-86.

14. Cojocaru M, Cojocaru IM, Silosi I, Vrabie CD. Manifestations of systemic lupus erythematosus. Maedica (Buchar). 2011;6(4):330-6. 
15. Lech $M$, Anders HJ. The pathogenesis of lupus nephritis. J Am Soc Nephrol. 2013;24(9):1357-66.

16. Simon JA, Cabiedes J, Ortiz E, Alcocer-Varela J, Sanchez-Guerrero J. Antinucleosome antibodies in patients with systemic lupus erythematosus of recent onset: potential utility as a diagnostic tool and disease activity marker. Rheumatology (Oxford). 2004;43(2):220-4.

17. Ho A, Magder LS, Barr SG, Petri M. Decreases in anti-double-stranded DNA levels are associated with concurrent flares in patients with systemic lupus erythematosus. Arthritis Rheum. 2001;44(10):2342-9.

18. Bigler C, Lopez-Trascasa M, Potlukova E, Moll S, Danner D, Schaller M, Trendelenburg M. Antinucleosome antibodies as a marker of active proliferative lupus nephritis. Am J Kidney Dis. 2008;51(4):624-9.

19. Marto N, Bertolaccini ML, Calabuig E, Hughes GR, Khamashta MA. Anti-C1d antibodies in nephritis: correlation between titres and renal disease activity and positive predictive value in systemic lupus erythematosus. Ann Rheum Dis. 2005;64(3):444-8.

20. Yang XW, Tan Y, Yu F, Zhao MH. Combination of anti-C1q and anti-dsDNA antibodies is associated with higher renal disease activity and predicts renal prognosis of patients with lupus nephritis. Nephrol Dial Transplant. 2012; 27(9):3552-9.

21. Nzeusseu Toukap A, Galant C, Theate I, Maudoux AL, Lories RJ, Houssiau FA, Lauwerys BR. Identification of distinct gene expression profiles in the synovium of patients with systemic lupus erythematosus. Arthritis Rheum. 2007;56(5):1579-88.

22. Leffler J, Martin M, Gullstrand B, Tyden H, Lood C, Truedsson L, Bengtsson AA, Blom AM. Neutrophil extracellular traps that are not degraded in systemic lupus erythematosus activate complement exacerbating the disease. J Immunol. 2012;188(7):3522-31.

23. Manzi S, Navratil JS, Ruffing MJ, Liu CC, Danchenko N, Nilson SE, Krishnaswami S, King DE, Kao AH, Ahearn JM. Measurement of erythrocyte C4d and complement receptor 1 in systemic lupus erythematosus. Arthritis Rheum. 2004;50(11):3596-604.

24. Liu CC, Manzi S, Kao AH, Navratil JS, Ruffing MJ, Ahearn JM. Reticulocytes bearing $\mathrm{C} 4 \mathrm{~d}$ as biomarkers of disease activity for systemic lupus erythematosus. Arthritis Rheum. 2005;52(10):3087-99.

25. Navratil JS, Manzi S, Kao AH, Krishnaswami S, Liu CC, Ruffing MJ, Shaw PS, Nilson AC, Dryden ER, Johnson JJ, et al. Platelet C4d is highly specific for systemic lupus erythematosus. Arthritis Rheum. 2006;54(2):670-4.

26. Kao AH, Navratil JS, Ruffing MJ, Liu CC, Hawkins D, McKinnon KM, Danchenko N, Ahearn JM, Manzi S. Erythrocyte C3d and C4d for monitoring disease activity in systemic lupus erythematosus. Arthritis Rheum. 2010;62(3):837-44.

27. Bergseth G, Ludviksen JK, Kirschfink M, Giclas PC, Nilsson B, Mollnes TE. An international serum standard for application in assays to detect human complement activation products. Mol Immunol. 2013;56(3):232-9.

\section{Submit your next manuscript to BioMed Central and we will help you at every step:}

- We accept pre-submission inquiries

- Our selector tool helps you to find the most relevant journal

- We provide round the clock customer support

- Convenient online submission

- Thorough peer review

- Inclusion in PubMed and all major indexing services

- Maximum visibility for your research

Submit your manuscript at www.biomedcentral.com/submit

CBiomed Central 